\title{
Religiosidade, espiritualidade e autoestima em adolescentes com fissura de lábio e palato: estudo correlacional*
}

\author{
Religiosity, spirituality, and self-esteem in adolescents with \\ cleft lip and palate: a correlational study \\ Religiosidad, espiritualidad y autoestima en adolescentes con \\ labio y paladar hendido: un estudio correlacional
}

Como citar este artigo:

Cunha GFM, Manso MMFG, Villlela MJCS, Bom GC, Mondini CCSD, Trettene AS. Religiosity, spirituality, and self-esteem in adolescents with cleft lip and palate: a correlational study. Rev Esc Enferm USP. 2021;55:e03782. https://doi.org/10.1590/S1980-220X2020030503782

\section{Gabriela Fávaro Marques da Cunha ${ }^{1}$ \\ Maila Meryellen Ferreira Garcia Manso ${ }^{1}$ \\ Maria Júlia Costa de Souza Villela ${ }^{1}$ \\ Gesiane Cristina Bom ${ }^{1}$ \\ Cleide Carolina da Silva Demoro Mondini ${ }^{1}$}

Armando dos Santos Trettene ${ }^{1}$

* Extraído da dissertação: "Religiosidade, espiritualidade e autoestima em adolescentes com fissura de lábio e palato uni e bilateral: estudo correlacional, Programa de Pós-Graduação em Ciências da Reabilitação, Hospital de Reabilitação de Anomalias Craniofaciais, Universidade de São Paulo, 2020.

${ }^{1}$ Universidade de São Paulo, Hospital de Reabilitação de Anomalias Craniofaciais, Programa de Pós-Graduação em Ciências da Reabilitação, Bauru, SP, Brasil.

\section{ABSTRACT}

Objective: To evaluate the correlation between religiosity, spirituality, and self-esteem in adolescents with uni- and bilateral cleft lip and palate. Method: Correlational and cross-sectional study developed in a public and tertiary hospital in Brazil between July 2018 and February 2019. The sample comprised 100 adolescents divided into two groups: G1 (unilateral cleft, 50 participants) and G2 (bilateral cleft, 50 participants). For data collection, three instruments were used: Sociodemographic Questionnaire, DUREL Religion index, Rosenberg self-esteem scale. Statistical analysis was performed with the tests Chi-squared, Mann-Whitney, Pearson Correlation, and analysis of linear correlation strength, with a 5\% significance level $(p \geq 0.05)$. Results: Only organizational religiosity was higher in $\mathrm{G} 1$ when compared with $\mathrm{G} 2(\mathrm{p}=0.03)$. The overall self-esteem was satisfactory for both groups; however, there was no significant difference between them $(p=0.34)$. No correlation between religiosity and spirituality with self-esteem were identified for G1 and G2. Conclusion: The adolescents with uni- or bilateral cleft lip and palate presented high levels of religiosity, spirituality, and self-esteem. However, no correlation between these variables was identified.

\section{DESCRIPTORS}

Religion; Spirituality; Self Concept; Adolescent; Cleft Lip; Cleft Palate. 


\section{INTRODUÇÃO}

Dentre as malformações craniofaciais, as fissuras de lábio e/ou palato são prevalentes, podendo acometer, isoladamente ou em associação, o lábio, rebordo alveolar e o palato. Sua etiologia é multifatorial e inclui fatores genéticos e ambientais. No Brasil, admite-se a incidência de um caso para cada 650 nascidos vivos ${ }^{(1-2)}$.

Pacientes acometidos por fissuras de lábio e/ou palato podem apresentar problemas funcionais (dificuldade no processo mastigação-deglutição-respiração, distúrbios dentários, audição, fonação, disfunções otológicas frequentes), estéticos e psicossociais (aparência física distinta, problemas de comunicação e socialização) que podem levar à exclusão social( ${ }^{(1)}$.

Inicialmente, os problemas enfrentados são funcionais, incluindo a alimentação. Posteriormente, em especial na adolescência, destacam-se os problemas estéticos e psicossociais $^{(3-4)}$. A adolescência compreende a idade entre os $10 \mathrm{e}$ os 19 anos, etapa em que o indivíduo aprende a administrar sentimentos e relacionamentos extrafamiliares ${ }^{(5)}$.

Adolescentes com fissura de lábio e/ou palato podem ser alvo de preconceito e discriminação, o que pode resultar em baixa autoestima, pouca aceitação social e isolamento. Contudo, esses comportamentos vão depender de sua história de vida, relações familiares, desenvolvimento de seu processo de reabilitação, padrões sociais e culturais ${ }^{(6)}$.

A adolescência é norteada por diversas transformações fisiológicas e psicológicas, as quais são influenciadas pela autoestima. $\mathrm{O}$ adolescente procura a todo instante a aprovação, tanto do contexto familiar, quanto do grupo social a que pertence. Nessa fase, é de suma importância refletir sobre os níveis de autoestima, além de analisar seus pensamentos e atitudes, pois influenciam diretamente o desempenho escolar e o convívio social ${ }^{(7)}$.

É comum haver um equívoco entre o conceito de autoimagem e o de autoestima. A autoimagem é como os outros veem o indivíduo, enquanto a autoestima corresponde a como o indivíduo vê a si mesmo. Portanto, a autoimagem gera a autoestima, ou seja, indivíduos com baixa autoimagem terão como consequência baixa autoestima ${ }^{(8)}$.

A autoestima em adolescentes com malformações pode apresentar-se comprometida, uma vez que as relações sociais são permeadas por sentimentos negativos, como a ansiedade, o medo e a vergonha. Nesse contexto, estratégias de enfrentamento ou coping são necessárias. Dentre elas, destaca-se a espiritualidade e/ou a religiosidade ${ }^{(9)}$.

Apesar de comumente serem utilizados como sinônimos, os termos religiosidade e espiritualidade se distinguem em suas definições. A religiosidade está vinculada à prática de uma religião ou doutrina específica, com suas crenças e adorações. Por outro lado, a espiritualidade refere-se a uma proporção humana mais ampla, considerando aquilo que dá sentido à vida e à existência, e nem sempre se encontra atrelada à religião ${ }^{(10)}$.

Estudos revelam que indivíduos, incluindo adolescentes, com altos níveis de religiosidade apresentam menores índices de consumo de drogas, vida sexual mais segura, sensação de bem-estar, melhor enfrentamento de crises, menor propensão a depressão e ansiedade, menores índices de suicídio e menor risco de desenvolver doenças cardiovasculares ${ }^{(11-14)}$.
Portanto, a religiosidade influencia a forma como a pessoa enfrenta situações de estresse, sofrimento e outros problemas da vida, podendo proporcionar-lhe maior aceitação, estabilidade e adaptação às situações difíceis enfrentadas, gerando paz, autoconfiança e imagem positiva de si mesmo, enquanto a espiritualidade, principalmente no caso de pessoas com alguma alteração física, pode levar a um melhor enfrentamento no processo reabilitador ${ }^{(11-14)}$.

Nesse sentido, um estudo que avaliou a correlação de espiritualidade e religiosidade com a qualidade de vida em adolescentes com fissura de lábio e/ou palato indicou que adolescentes mais espiritualizados demonstraram melhor percepção da qualidade de vida em comparação com os sem fissura ${ }^{(15)}$.

Ao que nos consta, até o momento, não há estudos que correlacionem a religiosidade e/ou a espiritualidade à autoestima em adolescentes, em particular naqueles com fissura de lábio e palato, reforçando a importância deste estudo de abordagem inédita.

Frente ao exposto, surgiram as seguintes questões: adolescentes com fissura de lábio e palato, uni ou bilateral, praticam mais a religiosidade e/ou são mais espiritualizados? Como se comporta a autoestima em adolescentes com fissura de lábio e palato? A autoestima dos adolescentes difere segundo a classificação da fissura (lábio e palato unilateral ou bilateral)? Existe correlação entre a religiosidade e/ou a espiritualidade em adolescentes com fissura de lábio e palato, uni e bilateral?

Propôs-se a hipótese de que adolescentes com fissura de lábio e palato unilateral, menos complexa anatomicamente em comparação com a bilateral, possuíam maiores níveis de religiosidade e espiritualidade, com influência positiva na autoestima.

Considerando-se a vulnerabilidade psicossocial dos adolescentes com fissura de lábio e palato, bem como os benefícios da espiritualidade e/ou da religiosidade como modalidade de coping, determinar sua correlação poderá fortalecer o uso dessas variáveis como indicadores de saúde e bem-estar.

Assim, o objetivo deste estudo foi avaliar a correlação entre religiosidade, espiritualidade e autoestima em adolescentes com fissura de lábio e palato, uni e bilateral.

\section{MÉTODO}

\section{Desenho do estudo}

Estudo descritivo, de correlação, transversal, de enfoque quantitativo, desenvolvido em um hospital público e terciário especializado no atendimento de pacientes com anomalias craniofaciais e síndromes relacionadas. A instituição conta com 91 leitos, sendo mantida com recursos do Sistema Único de Saúde e vinculada à Universidade de São Paulo. É reconhecida nacional e internacionalmente pelo atendimento interdisciplinar e humanizado na área assistencial, de ensino e de pesquisa.

\section{População}

A população foi composta por adolescentes com fissura de lábio e palato. Visando a comparação, formalizaram-se dois grupos a partir da classificação da fissura, sendo G1 composto por adolescentes com fissura de lábio e palato unilateral e G2 por adolescentes com fissura de lábio e palato bilateral. 


\section{CRITÉrios de SEleÇÃo}

Foram convidados a participar adolescentes que se encontraram em atendimento ambulatorial no hospital. Os critérios de inclusão foram: possuir idade entre 10 e 19 anos completos e ter sido submetido previamente às cirurgias primárias de queiloplastia (correção do lábio) e palatoplastia (correção do palato). Foram excluídos os adolescentes em uso de psicofármacos por estes alterarem a percepção.

\section{DeFINIÇÃO DA AMOSTRA}

Para o cálculo amostral, considerou-se um coeficiente de correlação de moderado $(0,4)$, erro de $5 \%$ e poder de teste de 80\%. Assim, estimaram-se 47 participantes em cada grupo. Por fim, optou-se por 50 participantes por grupo, ou seja, a mostra constou de 100 adolescentes.

\section{Coleta de Dados}

A coleta de dados foi realizada em ambiente privativo, individualmente, entre julho de 2018 e fevereiro de 2019. Inicialmente, foram informados aos participantes os objetivos da pesquisa; apresentou-se àqueles com idade inferior a 18 anos o Termo de Assentimento e a seus responsáveis legais, o Termo de Consentimento Livre e Esclarecido. Para os participantes com idade igual ou superior a 18 anos, foi apresentado o Termo de Consentimento Livre e Esclarecido. Ademais, foram aplicados os instrumentos de coleta de dados.

Foram utilizados três instrumentos: Questionário Sociodemográfico, a Escala de Religiosidade de Durel e a Escala de Autoestima de Rosenberg - EAR ${ }^{(16-17)}$.

O Questionário Sociodemográfico foi utilizado para caracterizar os participantes segundo as variáveis idade, sexo, escolaridade, estado civil/afetivo, classificação socioeconômica, religião, filhos e vínculo empregatício.

A Escala de Durel foi utilizada para avaliar a religiosidade e a espiritualidade. Esse instrumento engloba três dimensões da religiosidade: a religiosidade organizacional (RO), a não organizacional (RNO) e a intrínseca ou espiritualidade ${ }^{(16)}$. Assim, neste estudo, a religiosidade intrínseca foi referida como espiritualidade, em conformidade a literatura $^{(15-16,18-19)}$. Ressalta-se que a Durel aborda os principais preceitos da religiosidade, que inclui a espiritualidade ${ }^{(16,18,20)}$. De fato, embora a religiosidade e a espiritualidade possuam conceitos distintos, ambas se correlacionam e representam, portanto, uma construção ${ }^{(21)}$.

A RO refere-se à frequência em igrejas e/ou templos com interação social, variando o escore de um a seis. A RNO independe de outras pessoas. É a atividade religiosa individual (oração, meditação, prece) que apresenta escore de um a seis. A espiritualidade avalia o comportamento e a influência que a religião confere à vida do indivíduo e é composta de escore com pontuação variando de três a quinze. Para o cálculo do escore do instrumento, recomenda-se que os três domínios sejam analisados separadamente e não somados em um escore final ${ }^{(16)}$.

A Durel foi traduzida e validada para a população brasileira ${ }^{(20)}$. Em uma investigação que a utilizou para a população adolescente, o valor do Alfa de Cronbach foi de 0,82, acusando boa consistência interna ${ }^{(15)}$.
A EAR foi utilizada para avaliar a autoestima, constituindo-se como uma medida unidimensional, tipo Likert, com pontuação de um a quatro. É composta por dez questões que visam avaliar, de forma global, as atitudes positivas e negativas do indivíduo em relação a si próprio, dentre as quais seis questões remetem a uma visão positiva e quatro a uma visão negativa. A pontuação é de no mínimo um para concordo plenamente e máxima de quatro para discordo plenamente, com total entre 10 e 40 pontos. Quanto maior o escore, maior será o nível de autoestima. Um nível de autoestima satisfatório tem escore igual ou maior a 30 pontos $^{(17)}$.

Os dados referentes à caracterização sociodemográfica foram obtidos através de consulta a fonte secundária de dados, ou seja, por consulta ao prontuário. Tanto a Escala de Durel quanto a EAR são autoaplicáveis. O tempo médio de coleta de dados foi de 30 minutos.

\section{ANÁlISE E TRATAMENTO DOS DADOS}

Para comparar os grupos quanto às características sociodemográficas (sexo, escolaridade, estado civil, classificação socioeconômica, religião e vínculo empregatício), utilizou-se o Teste Qui-Quadrado. Na comparação entre os níveis de religiosidade, espiritualidade e autoestima entre os grupos, utilizou-se o Teste Mann-Whitney. Visando correlacionar as medidas de interesse, religiosidade, espiritualidade e autoestima em ambos os grupos, foi aplicado o teste de Correlação de Pearson. Ainda, foi utilizada a análise das forças de correlação linear entre as medidas, a qual determina que valores de correlação menores do que 0,30 indicam fraca correlação, ou seja, mesmo quando estatisticamente significantes não apresentam relevância clínica; valores entre 0,30 e 0,50 evidenciam moderada correlação e acima de 0,50 demonstram forte correlação ${ }^{(22)}$. O nível de significância adotado para todos os testes foi de $5 \%(\mathrm{p} \leq 0,05)$.

\section{Aspectos ÉtICOS}

A coleta de dados iniciou-se após a aprovação do projeto pelo Comitê de Ética em Pesquisa em Seres Humanos do Hospital por meio do Parecer Consubstanciado 2.739.764 de 2018 e CAAE 87138618.3.0000.5441. Os participantes com idade inferior a 18 anos formalizaram sua participação por meio da assinatura do Termo de Assentimento, enquanto seus responsáveis legais consentiram assinando o Termo de Consentimento Livre e Esclarecido, concomitantemente. Os participantes com idade igual ou superior a 18 anos assinaram o Termo de Consentimento Livre e Esclarecido, em consonância com a Resolução no 466/2012 do Conselho Nacional de Saúde.

\section{RESULTADOS}

Participaram do estudo 100 adolescentes, divididos em dois grupos, G1 e G2, sendo G1 composto por 50 adolescentes com fissura de lábio e palato unilateral e G2 por 50 adolescentes com fissura de lábio e palato bilateral. No G1 a média de idade foi de 15,5 anos ( $\mathrm{DP}=2,7)$, enquanto no G2 a média foi de 14,6 anos ( $\mathrm{DP}=2,5)$. 
No G1 prevaleceram participantes do sexo masculino $(\mathrm{n}=36 ; 72 \%)$, com ensino médio incompleto $(\mathrm{n}=20 ; 40 \%)$, solteiros ( $n=43 ; 86 \%)$, com classificação socioeconômica baixa superior $(n=33 ; 66 \%)$, protestantes $(n=21 ; 42 \%)$ e sem vínculo empregatício $(\mathrm{n}=39 ; 78 \%)$. No G2 predominaram participantes do sexo masculino $(n=34 ; 72 \%)$, com ensino fundamental completo $(\mathrm{n}=28 ; 56 \%)$, solteiros ( $n=42 ; 84 \%)$, com classificação socioeconômica baixa superior $(\mathrm{n}=35 ; 70 \%)$, católicos $(\mathrm{n}=22 ; 44 \%)$ e sem vínculo empregatício $(n=42 ; 84 \%)$. Não foram observadas diferenças estatísticas significantes em relação à caracterização sociodemográfica entre os grupos, conforme a Tabela 1.

Tabela 1 - Distribuição dos participantes segundo as características sociodemográficas sexo, escolaridade, estado civil, classificação socioeconômica, religião e vínculo empregatício- Bauru, SP, Brasil, 2019.

\begin{tabular}{|c|c|c|c|c|c|c|}
\hline \multirow{2}{*}{ Variável } & & \multicolumn{2}{|c|}{ G1 } & \multicolumn{2}{|c|}{ G2 } & \multirow{2}{*}{ Valor de $p$} \\
\hline & & $\mathbf{N}$ & $\%$ & $\mathbf{N}$ & $\%$ & \\
\hline \multirow{2}{*}{ Sexo* } & Masculino & 36 & 72 & 34 & 68 & \multirow{2}{*}{0,82} \\
\hline & Feminino & 14 & 28 & 16 & 32 & \\
\hline \multirow{4}{*}{ Escolaridade* $^{*}$} & Ensino Superior Incompleto & 5 & 10 & 1 & 2 & \multirow{4}{*}{0,09} \\
\hline & Ensino Médio Completo & 6 & 12 & 2 & 4 & \\
\hline & Ensino Médio Incompleto & 20 & 40 & 19 & 38 & \\
\hline & Ensino Fundamental Incompleto & 19 & 38 & 28 & 56 & \\
\hline \multirow{3}{*}{ Estado Civil/afetivo* } & Solteiro & 43 & 86 & 42 & 84 & \multirow{3}{*}{0,60} \\
\hline & Namorando & 7 & 14 & 7 & 14 & \\
\hline & Casado & - & - & 1 & 2 & \\
\hline \multirow{4}{*}{ Classificação socioeconômica* } & Baixa Inferior & 9 & 18 & 8 & 16 & \multirow{4}{*}{0,77} \\
\hline & Baixa Superior & 33 & 66 & 35 & 70 & \\
\hline & Média Inferior & 7 & 14 & 7 & 14 & \\
\hline & Média & 1 & 2 & - & - & \\
\hline \multirow{4}{*}{ Religião* } & Protestante & 21 & 42 & 21 & 42 & \multirow{4}{*}{0,35} \\
\hline & Católico & 20 & 40 & 22 & 44 & \\
\hline & Ateu/Agnóstico/Irreligião & 9 & 18 & 5 & 10 & \\
\hline & Espírita & - & - & 2 & 4 & \\
\hline \multirow{2}{*}{ Vínculo empregatício* } & Não & 39 & 78 & 42 & 84 & \multirow{2}{*}{0,61} \\
\hline & Sim & 11 & 22 & 8 & 16 & \\
\hline
\end{tabular}

Nota: $(n=100)$

* Teste Qui-Quadrado; nível de significância adotado de 5\% ( $\leq 0,05)$

Em relação à $\mathrm{RO}, \mathrm{RNO}$ e à espiritualidade, evidenciou-se que apenas a $\mathrm{RO}$ foi maior no G1 em comparação com o G2 $(p=0,03)$ (Tabela 2). Contudo, observou-se que os valores de mediana apresentaram-se elevados em ambos os grupos para todas as variáveis estudadas, conforme demonstrado na Tabela 2.

Tabela 2 - Distribuição dos participantes segundo os níveis de Religiosidade Organizacional (RO), Religiosidade Não Organizacional (RNO) e espiritualidade - Bauru, SP, Brasil, 2019.

\begin{tabular}{|c|c|c|c|c|c|c|c|c|}
\hline Variável & & $\mathbf{N}$ & Mediana & Q1§ & Q3|| & Média & Desvio padrão & Valor de $p$ \\
\hline \multirow{2}{*}{ Religiosidade Organizacional† } & G1 & 50 & 2,0 & 1,0 & 4,0 & 2,5 & 1,8 & \multirow{2}{*}{$0,03 \neq$} \\
\hline & $\mathrm{G} 2$ & 50 & 3,0 & 2,0 & 4,0 & 3,0 & 1,6 & \\
\hline \multirow{2}{*}{ Religiosidade Não Organizacional† } & G1 & 50 & 2,0 & 2,0 & 5,0 & 3,0 & 1,7 & \multirow{2}{*}{0,11} \\
\hline & $\mathrm{G} 2$ & 50 & 3,0 & 2,0 & 5,0 & 3,5 & 1,8 & \\
\hline \multirow{2}{*}{ Espiritualidadet } & G1 & 50 & 5,0 & 4,0 & 8,0 & 6,1 & 3,0 & \multirow{2}{*}{0,64} \\
\hline & G2 & 50 & 5,0 & 4,0 & 8,0 & 5,7 & 2,4 & \\
\hline
\end{tabular}

Nota: $(\mathrm{n}=100)$

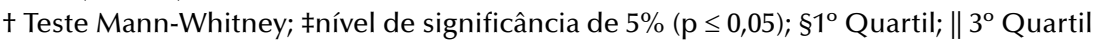

Evidenciou-se que a autoestima geral foi satisfatória em ambos os grupos (32 pontos no G1 e 30 pontos no G2). No entanto, não se observou diferença estatística significante conforme a Tabela 3 . 
Tabela 3 - Distribuição dos participantes segundo os níveis de Autoestima Positiva, Negativa e Geral nos G1 e G2 - Bauru, SP, Brasil, 2019.

\begin{tabular}{|c|c|c|c|c|c|c|c|c|}
\hline Variável & & $\mathrm{N}$ & Mediana & Q1§ & Q3|| & Média & Desvio padrão & Valor de $p$ \\
\hline \multirow{2}{*}{ Autoestima positivat } & G1 & 50 & 17,0 & 15,0 & 18,0 & 16,3 & 2,3 & \multirow{2}{*}{0,18} \\
\hline & G2 & 50 & 15,5 & 13,8 & 18,0 & 15,5 & 2,8 & \\
\hline \multirow{2}{*}{ Autoestima negativa +} & G1 & 50 & 16,0 & 11,8 & 18,0 & 14,6 & 3,9 & \multirow{2}{*}{0,92} \\
\hline & G2 & 50 & 14,5 & 11,0 & 18,0 & 14,3 & 4,4 & \\
\hline \multirow{2}{*}{ Autoestima geralt } & G1 & 50 & 32,0 & 27,0 & 35,0 & 31,1 & 5,3 & \multirow{2}{*}{0,34} \\
\hline & G2 & 50 & 30,0 & 24,8 & 35,0 & 29,7 & 6,5 & \\
\hline
\end{tabular}

Nota: $(\mathrm{n}=100)$

† Teste Mann-Whitney; nível de significância de 5\% (p $\leq 0,05)$; §1 Quartil; || $3^{\circ}$ Quartil

Não foram evidenciadas correlações entre a RO, RNO e a espiritualidade com a autoestima no G1 (Tabela 4).

Tabela 4 - Correlação entre Religiosidade Organizacional, Religiosidade Não Organizacional, Espiritualidade e Autoestima em pacientes com fissura de lábio e palato unilateral (G1) - Bauru, SP, Brasil, 2019.

\begin{tabular}{lccc}
\hline Variável de correlação & $\mathbf{r}$ & Correlação & Valor de $\mathbf{p}$ \\
\hline Religiosidade Organizacional $\boldsymbol{\Phi}$ & & & \\
Autoestima positiva & $-0,12$ & Fraca & 0,39 \\
Autoestima negativa & $-0,10$ & Fraca & 0,48 \\
Autoestima geral & $-0,12$ & Fraca & 0,39 \\
\hline Religiosidade Não Organizacional & & & \\
Autoestima positiva & $-0,23$ & Fraca & 0,10 \\
Autoestima negativa & $-0,20$ & Fraca & 0,17 \\
Autoestima geral & $-0,23$ & Fraca & 0,24 \\
\hline Espiritualidade & & & \\
Autoestima positiva & $-0,23$ & Fraca & 0,10 \\
Autoestima negativa & $-0,03$ & Fraca & 0,82 \\
Autoestima geral & $-0,11$ & Fraca & 0,44 \\
\hline Nota: $(\boldsymbol{n}$ & &
\end{tabular}

Nota: $(\mathrm{n}=100)$

ๆ Teste Correlação de Pearson; nível de significância de $5 \%(p \leq 0,05)$

\section{DISCUSSÃO}

Por muito tempo, as questões que abordavam a religiosidade foram depreciadas no meio científico por serem consideradas áreas opostas e difíceis de serem controladas. Contudo, na atualidade, as experiências relacionadas à religiosidade vêm sendo notadas como importantes elementos da vida dos indivíduos, despertando interesse entre pesquisadores e sendo incorporado aos sistemas de saúde ${ }^{(11)}$.

No presente estudo, no âmbito religioso e espiritual, observou-se predomínio da $\mathrm{RO}$ nos adolescentes com fissura de lábio e palato unilateral, em comparação aos adolescentes com a malformação bilateral. Esses resultados demonstram que adolescentes com fissuras unilaterais frequentavam mais encontros religiosos, tais como: templos, missas, cultos, cerimônias, grupos de estudo religioso, grupos de oração, entre outros ${ }^{(16)}$.

A RO relaciona-se diretamente à interação e apoio social, tão necessários aos adolescentes com fissura, considerando-se as implicações sociais que experienciam. Adolescentes com fissuras unilaterais, pelo fato de apresentarem menor comprometimento anatômico, funcional e estético, apresentam maior facilidade de interação social.
Não foram evidenciadas correlações entre a RO, RNO e a espiritualidade com a autoestima no G2 (Tabela 5).

Tabela 5 - Correlação entre Religiosidade Organizacional, Religiosidade Não Organizacional, Espiritualidade e Autoestima, em pacientes com fissura de lábio e palato bilateral (G2) - Bauru, SP, Brasil, 2019.

\begin{tabular}{lccc}
\hline Variáveis de correlação & $\mathbf{r}$ & Correlação & Valor de $\mathbf{p}$ \\
\hline Religiosidade Organizacional $\mathbf{q}$ & \multicolumn{3}{|}{} \\
Autoestima positiva & $-0,14$ & Fraca & 0,32 \\
Autoestima negativa & $-0,10$ & Fraca & 0,48 \\
Autoestima geral & $-0,15$ & Fraca & 0,29 \\
\hline Religiosidade Não Organizacional & & & \\
Autoestima positiva & $-0,31$ & Fraca & 0,06 \\
Autoestima negativa & $-0,10$ & Fraca & 0,47 \\
Autoestima geral & $-0,21$ & Fraca & 0,14 \\
\hline Espiritualidade & & & \\
Autoestima positiva & $-0,24$ & Fraca & 0,09 \\
Autoestima negativa & $-0,21$ & Fraca & 0,15 \\
Autoestima geral & $-0,26$ & Fraca & 0,07 \\
\hline
\end{tabular}

Nota: $(\mathrm{n}=100)$

ๆ Teste Correlação de Pearson; nível de significância de 5\% (p $\leq 0,05)$

Frente à realidade social segmentada, que inviabiliza o acesso a serviços públicos de saúde de forma equânime, destaca-se a importância das interações sociais e de inclusão em comunidades, grupos e redes de apoio tanto para enfrentamento situacional como financeiro, independentemente de serem compostas por familiares, amigos, vizinhos, instituições religiosas, entre outras ${ }^{(23)}$.

Portanto, melhorar o acesso aos cuidados é fundamental para atingir efetivamente as metas de atendimento e reabilitação de pacientes com fissuras de lábio e/ou palato, incluindo suas famílias. Ressalta-se ainda a importância de acompanhar pacientes com fissura da adolescência até a idade adulta para explorar as trajetórias de desenvolvimento psicossocial ${ }^{(24)}$.

Conforme evidenciado neste estudo, os adolescentes com fissura, em geral, pertenciam à classe social menos favorecida. Em outras palavras, pertencer a essa condição social indica que esses indivíduos dispõem de uma infraestrutura de moradia, nível de escolaridade e faixa salarial desfavorá$\mathrm{vel}^{(6)}$. Assim, infere-se que por tal motivo esses adolescentes buscam apoio psicossocial na RO. De fato, adolescentes com fissura apresentam maiores níveis de RO em comparação àqueles sem a malformação ${ }^{(15)}$. 
Estar inserido em grupos sociais e possuir amigos reforça sentimentos de aceitação e interação social, além de minimizar sensações negativas e preocupações relacionadas à aparência física. Desse modo, o incentivo dessas práticas é recomendado como parte das intervenções terapêuticas ${ }^{(25)}$.

Quanto à RNO, embora sem diferença estatística entre adolescentes com fissuras uni ou bilaterais, os escores foram altos para ambos, indicando o hábito de praticar a religiosidade de maneira mais íntima através de orações, meditação, ouvindo ou assistindo programas religiosos, com leitura de livros e/ou textos religiosos, entre outros ${ }^{(16)}$.

De fato, um estudo que incluiu adolescentes com diagnóstico de câncer demonstrou que a maioria deles considerava que as orações lhes proporcionavam sensação de calma, tranquilidade e aumento da confiança, influenciando positivamente o tratamento e a recuperação da saúde ${ }^{(23)}$.

Altos níveis de $\mathrm{RNO}$ vinculam-se à $\mathrm{RO}$, ou seja, o indivíduo tem a oportunidade de colocar em prática sua crença de maneira individualizada ${ }^{(16)}$. Nesse sentido, estudo realizado com adolescentes com fissura de lábio e/ou palato retratou a correlação entre os níveis de RO e RNO, demonstrando a interligação entre elas ${ }^{(15)}$.

Em relação à espiritualidade, apesar de não ter sido observada diferença estatística significante entre os adolescentes com fissura unilateral em comparação aos com fissura bilateral, ambos apresentaram escores elevados, indicando que esses adolescentes buscavam um significado ou sentido para a vivência humana, tendo relação ou não com uma religião ${ }^{(16)}$.

Estudo realizado com adolescentes sem fissura, com o objetivo de verificar a influência da espiritualidade nos relacionamentos interpessoais, apontou que a espiritualidade exerceu grande influência na convivência com a família, amigos, nas relações amorosas e na vida. Ainda, destacou-se o aumento da proximidade, respeito e fortalecimento da união no contexto familiar. Evidenciou-se o aumento do número de amigos e preocupação em evitar discussões. Por fim, quanto aos relacionamentos amorosos, observou-se a busca de parceiros com as mesmas crenças ou religião ${ }^{(26)}$.

Uma investigação que incluiu adolescentes com fissura de lábio e/ou palato evidenciou correlação entre a espiritualidade e uma melhor percepção da qualidade de vida ${ }^{(15)}$. Outro estudo que incluiu adolescentes iranianos revelou que estes utilizavam crenças, ensinamentos e experiências espirituais para controlar o estresse, gerenciar crises e lidar com problemas em geral ${ }^{(27)}$.

Em relação aos níveis de autoestima geral, observou-se que foi satisfatório tanto no grupo dos adolescentes com fissura de lábio e palato unilateral quanto no grupo com fissura bilateral. Considera-se que uma pessoa com autoestima elevada retém uma imagem contínua das suas capacidades, com maior oportunidade de assumir comportamentos ativos em grupos sociais, além de tender a orientar-se de maneira direta e ativa em busca de suas metas pessoais ${ }^{(7)}$.

Infere-se que os elevados níveis de autoestima evidenciados nos adolescentes participantes deste estudo relacionaram-se à sua reabilitação em um centro de excelência, o que certamente influenciou os resultados estéticos e funcionais. A satisfação do paciente com o resultado do tratamento é de extrema importância e influencia a percepção de si próprio e do meio em que vive. Contudo, a aparência facial, incluindo a nasal e labial em pacientes com fissura operada, não é o único fator influenciador da autoestima, ou seja, fatores psicossociais apresentam igual relevância ${ }^{(28)}$.

Em contrapartida, diferentes estudos demonstraram que adolescentes com fissura apresentam baixa autoestima ${ }^{(29-30)}$. Pesquisa concluiu que adolescentes com fissura de lábio e/ou palato, reparadas, apresentaram baixa autoestima associada a supostos defeitos, manchas, cicatrizes, problemas de estética nasal e dentários, quando comparados a adolescentes sem fissuras ${ }^{(29)}$.

Estudo indicou que adolescentes com fissura de lábio e/ou palato apresentaram baixa autoestima em comparação aos adolescentes sem fissura, relacionando-se a insatisfação com a voz, que gerou problemas de comunicação, e insatisfação com a estética facial, incluindo o lábio, nariz e/ou dentes, ocasionando fragilidade psicoemocional ${ }^{(30)}$.

De fato, percepções estéticas, dentre elas as referentes ao contexto ortodôntico, influenciam o autoconceito psicológico do paciente, a confiança social e a necessidade de tratamento. Esses aspectos apresentam maior relevância à medida que $\mathrm{O}$ paciente amadurece, ou seja, na adolescência e na idade adulta ${ }^{(31)}$.

Estudo australiano que avaliou a autoestima e os fatores a ela relacionados em adolescentes com e sem fissura de lábio e/ou palato mostrou que possuir fissuras mais complexas anatomicamente, estar acima do peso e dar alta importância à aparência facial foram determinantes para baixa autoestima, enquanto manter um peso corporal normal e atribuir menor importância à aparência facial contribuiu para melhorá- $l a^{(32)}$.

Ressalta-se, porém, que possuir altos níveis de autoestima não significa necessariamente que os adolescentes não enfrentarão outros problemas de ordem psicossocial. Nesse contexto, uma investigação que incluiu adolescentes noruegueses concluiu que, embora possuir fissura de lábio e/ou palato tivesse pouco impacto sobre os sintomas depressivos e de baixa autoestima, a prevalência de relacionamentos românticos foi significativamente menor entre os adolescentes com fissura de lábio e/ou palato em comparação àqueles sem a malformação(25).

Ademais, outra pesquisa que avaliou, entre outros objetivos, a autoestima de adolescentes e adultos poloneses, com e sem fissura de lábio e/ou palato, mostrou não existir diferença significante entre eles ${ }^{(33)}$. Em suma, esses achados indicam a complexidade do autoconceito e dos fatores que influenciam a autoestima.

Contrariando a hipótese inicial desta investigação, não se evidenciou correlação entre a religiosidade, espiritualidade e a autoestima. A espiritualidade e/ou a religiosidade são conceitos amplos e dinâmicos que podem influenciar significados ou percepções, o que se acredita ter influenciado esse resultado. Soma-se a isso que os adolescentes podem replicar valores absorvidos por influência dos contextos espirituais e religiosos de seus pais, sem que necessariamente os tenham incorporados ou os vivenciem como deveriam. Por isso, podem usufruir parcialmente de seus benefícios ${ }^{(15)}$.

Ressalta-se que os níveis de autoestima se relacionam a fatores intrínsecos e extrínsecos. Por consequência, o apoio familiar e o processo reabilitador são de extrema importância, não só voltados aos aspectos estéticos, mas também aos aspectos psicológico, físico, social e funcional desses 
adolescentes. Após a correção cirúrgica da malformação, evidenciam-se os benefícios quanto à melhora nos níveis de autoestima e autoconfiança, que contribuem para melhor percepção da qualidade de vida.

Por fim, a característica monocêntrica e o desenho transversal deste estudo configuram-se como limitações, uma vez que não permitem avaliar relações de causa e efeito. Assim, estudos multicêntricos e prospectivos são encorajados.

No entanto, os benefícios deste estudo são evidentes e incluem uma investigação detalhada sobre a religiosidade, a espiritualidade e a autoestima em adolescentes com fissura de lábio e palato, uni ou bilaterais. Embora a correlação entre as variáveis estudadas não tenha sido evidenciada, o estudo possibilitou identificar que os adolescentes deste estudo apresentaram elevados níveis de religiosidade, espiritualidade e de autoestima.

\section{CONCLUSÃO}

Adolescentes com fissura de lábio e palato, uni ou bilateral, apresentaram elevados níveis de religiosidade, espiritualidade e de autoestima. Entretanto, contrariando a hipótese, não se evidenciou correlação entre as variáveis estudadas.

\section{RESUMO}

Objetivo: Avaliar a correlação entre religiosidade, espiritualidade e autoestima em adolescentes com fissura de lábio e palato, uni e bilateral. Método: Estudo correlacional e transversal, desenvolvido em um hospital público e terciário brasileiro, entre julho de 2018 e fevereiro de 2019. A amostra constou de 100 adolescentes divididos em dois grupos, G1 (fissura unilateral, 50 participantes) e G2 (fissura bilateral, 50 participantes). Para a coleta de dados, foram utilizados três instrumentos: Questionário Sociodemográfico, a Escala de Religiosidade de Durel e a Escala de Autoestima de Rosenberg. Utilizaram-se para a análise estatística os Testes Qui-Quadrado, Mann-Whitney, Correlação de Pearson e a análise das forças de correlação linear, com nível de significância de 5\% ( $p \geq 0,05)$. Resultados: Apenas a religiosidade organizacional foi maior no $\mathrm{G} 1 \mathrm{em}$ comparação com o $\mathrm{G} 2(\mathrm{p}=0,03)$. A autoestima geral foi satisfatória em ambos os grupos; contudo, não houve diferença significante entre eles $(p=0,34)$. Não foram evidenciadas correlações de religiosidade e espiritualidade com a autoestima nos G1 e G2. Conclusão: Adolescentes com fissura de lábio e palato, uni ou bilateral, apresentaram elevados níveis de religiosidade, espiritualidade e autoestima. Porém, não se evidenciou correlação entre essas variáveis.

\section{DESCRITORES \\ Religião; Espiritualidade; Autoimagem; Adolescente; Fenda Labial; Fissura Palatina.}

\section{RESUMEN}

Objetivo: Evaluar la correlación entre religiosidad, espiritualidad y autoestima en adolescentes con labio y paladar hendido unilateral y bilateral. Método: Estudio correlacional y transversal desarrollado en un hospital público y terciario de Brasil entre julio de 2018 y febrero de 2019. La muestra consistió en 100 adolescentes divididos en dos grupos, G1 (fisura unilateral, 50 participantes) y G2 (fisura bilateral, 50 participantes). Para la recogida de datos se utilizaron tres instrumentos: Cuestionario sociodemográfico, Escala de Religiosidad DUREL y Escala de Autoestima de Rosenberg. Para el análisis estadístico se utilizaron los testes de Chi-cuadrado, Mann-Whitney, correlación de Pearson y el análisis de la fuerza de correlación lineal, con un nivel de significación del 5\% ( $p \geq 0,05)$. Resultados: Sólo la religiosidad organizacional fue mayor en el G1 en comparación con el G2 ( $p=0,03$ ). La autoestima general fue satisfactoria en ambos grupos, pero no hubo diferencias significativas entre ellos $(p=0,34)$. No se evidenciaron correlaciones de religiosidad y espiritualidad con la autoestima en G1 y G2. Conclusión: Los adolescentes con labio y paladar hendido unilateral o bilateral presentan elevados niveles de religiosidad, espiritualidad y autoestima. Sin embargo, no se evidenció una correlación entre estas variables.

\section{DESCRIPTORES}

Religión; Espiritualidad; Autoimagen; Adolescente; Labio Leporino; Fissura del Paladar.

\section{REFERÊNCIAS}

1. Freitas JA, Neves LT, Almeida AL, Garib DG, Trindade-Suedam IK, Yaedú RY, et al. Rehabilitative treatment of cleft lip and palate: experience of the Hospital for Rehabilitation of Craniofacial Anomalies/USP (HRAC/USP)—Part 1: overall aspects. J Appl Oral Sci. 2012;20(1):9-15. https://doi.org/10.1590/S1678-77572012000100003

2. Xiao Y, Taub MA, Ruczinski I, Begum F, Hetmanski JB, Schwender H, et al. Evidence for SNP-SNP interaction identified through targeted sequencing of cleft case-parent trios. Genet Epidemiol. 2017;41(3):244-50. https://doi.org/10.1002/gepi.22023

3. Cunha GF, Mondini CC, Almeida RJ, Bom GC. Prenatal discovery of baby's cleft lip and palate: pregnant women's main doubts. Rev Enferm UERJ. 2019;27:e34127. https://doi.org/10.12957/reuerj.2019.34127

4. Hlongwa P, Rispel LC. "People look and ask lots of questions": caregivers' perceptions of healthcare provision and support for children born with cleft lip and palate. BMC Public Health. 2018;18(1):506. https://doi.org/10.1186/s12889-018-5421-x

5. World Health Organization. Adolescent development [Internet]. Geneva: World Health Organization; 2018 [cited 2019 Jan 3]. Available from: http://www.who.int/maternal_child_adolescent/topics/adolescence/dev/en/\#

6. Graciano MI, Souza EG, Rosa JA, Blattner SH. [Content validation of an instrument of socio-economic assessment whitin the social servisse]. RIPE - Rev Inst Pesq Estud Constr Serv Soc [Internet]. 2015 [cited 2020 Jun 9];19(36):29-57. Portuguese. Available from: http://ojs.ite.edu.br/index.php/css/article/viewFile/214/253

7. Deffendi LT, Schelini PW. [Self-esteem, overall intellectual level and metacognitive knowledge in teenagers]. Psicol Esc Educ. 2014;18(2):313-20. Portuguese. https://doi.org/10.1590/2175-3539/2014/0182752.

8. Jesus PB, Santos I, Brandão ES. Self-image and self-esteemin persons with skin disorders:an integrative literature review based Callista Roy's model. Aquichan. 2015;15(1):75-89. Portuguese. https://doi.org/10.5294/aqui.2015.15.1.8

9. Taheri Kharame Z, Zamanian H, Foroozanfar S, Afsahi S. Religious wellbeing as a predictor for quality of life in Iranian hemodialysis patients. Glob J Health Sci. 2014;6(4):261-9. https://doi.org/10.5539/gjhs.v6n4p261 
10. Machado FR, Zangari W, Maraldi EO, Martins LB, Shimabucuro AH. Contribuições da psicologia para a compreensão das relações entre a espiritualidade, a religiosidade e as experiências anômalas. Clareira Rev Filosof Reg Amazônica [Internet]. 2015 [cited 2020 Jun 9];3(2):1-21. Available from: http://www.revistaclareira.com.br/index.php/clareira/article/view/114

11. Santero M, Daray FM, Prado C, Hernández-Vásquez A, Irazola V. Association between religiosity and depression varies with age and sex among adults in South America: evidence from the CESCAS I study. PLoS One. 2019;14(12):e0226622. https://doi.org/10.1371/journal. pone.0226622

12. Kent BV, Stroope S, Kanaya AM, Zhang Y, Kandula NR, Shields AE. Private religion/spirituality, self-rated health, and mental health among US South Asians. Qual Life Res. 2020;29(2):495-504. https://doi.org/10.1007/s11136-019-02321-7

13. Michaelson V, King N, Inchley J, Currie D, Brooks F, Pickett W. Domains of spirituality and their associations with positive mental health: a study of adolescents in Canada, England and Scotland. Prev Med. 2019;125:12-8. https://doi.org/10.1016/j.ypmed.2019.04.018

14. Ozdemir A, Saritas S. Effect of yoga nidra on the self-esteem and body image of burn patients. Complement Ther Clin Pract. 2019;35:86-91. https://doi.org/10.1016/j.ctcp.2019.02.002

15. Farinha FT, Banhara FL, Bom GC, Kostrisch LM, Prado PC, Trettene AS. Correlation between reliogiosity, spirituality and quality of life in adolescents with and without cleft lip and palate. Rev Latino-Am Enfermagem. 2018;26:e3059. https://doi.org/10.1590/1518-8345.2498-3059

16. Moreira-Almeida A, Peres MF, Aloe F, Lotufo Neto F, Koenig H. Portuguese version of Duke Religious Index - DUREL. Rev Psi Clin. 2008 [cited 2020 Jun 9];35(1):31-2. Available from: https://www.scielo.br/pdf/rpc/v35n1/v35n1a06.pdf

17. Sbicigo JB, Bandeira DR, Dell'Aglio DD. [Rosenberg Self-Esteem Scale (RSS): factorial validity and internal consistency]. Psico-USF. 2010;15(3):395-403. Portuguese. https://doi.org/10.1590/S1413-82712010000300012

18. Lucchetti G, Lucchetti ALG, Peres MF, Leão FC, Moreira-Almeida A, Koenig HG. Validation of the Duke Religion Index: DUREL (Portuguese version). J Relig Health. 2012;51(2):579-86. https://doi.org/10.1007/s10943-010-9429-5

19. Bravin AM, Trettene AS, Cavalcante RS, Banin VB, Padula NA, Saranholi TL, et al. Influence of spirituality on renal function of kidney transplant patients. Acta Paul Enferm. 2017;30(5):504-11. https://doi.org/10.1590/1982-0194201700073

20. Cruz JP, Colet PC, Qubeilat H, Al-Otaibi J, Coronel El, Suminta RC. Religiosity and health-related quality of life: a cross-sectional study on filipino christian hemodialysis patients. J Relig Health. 2016;55(3):895-908. https://doi.org/10.1007/s10943-015-0103-9

21. Taunay TC, Gondim FA, Macêdo DS, Moreira-Almeida A, Gurgel LA, Andrade LM, et al. Validação da versão brasileira da escala de religiosidade de Duke (DUREL). Rev Psiq Clín. 2012;39(4):130-5. https://doi.org/10.1590/S0101-60832012000400003

22. Mukaka MM. Statistics corner: a guide to appropriate use of correlation coefficient in medical research. Malawi Med J [Internet]. 2012 [cited 2020 Jun 9];24(3):69-71. Available from: https://www.ncbi.nlm.nih.gov/pmc/articles/PMC3576830/

23. Souza VM, Frizzo HC, Paiva MH, Bousso RS, Santos AS. Spirituality, religion and personal beliefs of adolescents with câncer. Rev Bras Enferm. 2015;68(5):509-14. https://doi.org/10.1590/0034-7167.2015680504i

24. Bennett KG, Ranganathan K, Patterson AK, Baker MK, Vercler CJ, Kasten SJ, et al. Caregiver-reported outcomes and barriers to care among patients with cleft lip and palate. Plast Reconstr Surg. 2018;142(6):884e-91e. https://doi.org/10.1097/PRS.0000000000004987

25. Feragen KB, Stock NM, Sharratt ND, Kvalem IL. Self-perceptions of romantic appeal in adolescents with a cleft lip and/or palate. Body Image. 2016;18:143-52. https://doi.org/10.1016/j.bodyim.2016.06.009

26. Drosdek LP, Geronasso MC. [The influence of spirituality in interpersonal relationships of teenagers who are engaged in the young apprentice program]. Rev. Psicol Foco [Internet]. 2015 [cited 2020 Jun 9];7(10):35-53. Portuguese. Available from: http://revistas.fw.uri. br/index.php/psicologiaemfoco/article/view/2027

27. Hekmati Pour N, Mahmoodi-Shan GR, Ebadi A, Behnampour N. Spiritual self-care in adolescents: a qualitative study. Int J Adolesc Med Health. 2020 Oct 16 Ahead of print. https://doi.org/10.1515/ijamh-2019-0248

28. Patjanasoontornm N, Wongniyom K, Pradubwong S, Piyavhakul N, Chowchuen B. A Relationship between nasolabial appearance and selfesteem in adolescent with repaired cleft lip and cleft palate at Khon Kaen University Cleft Center. J Med Assoc Thai [Internet]. 2014 [cited 2020 Jun 9];97(10 Suppl 10):S49-52. Available from: https://kkucleft.kku.ac.th/research/JMAT_FINAL/JMAT\%20Final\%202014/JMAT\%20 2014\%20Vol\%2097,\%20No\%2010-7.pdf

29. Andrade D, Angerami EL. [The self-esteem of adolescents with and without a cleft lip and/or palate]. Rev Latino-Am Enfermagem. 2001;9(6):37-41. Portuguese. https://doi.org/10.1590/S0104-11692001000600007

30. Glaeser A, Costa SS, Collares MV. Cleft lip and palate: evaluation of the psycological impact using the Rosenberg self-esteem scale. Rev Bras Cir Plást. 2018;33(2):187-95. https://doi.org/10.5935/2177-1235.2018RBCP0094

31. Raghavan S, Philip K, Batra P, Marcusson A. Aesthetic perceptions and psychosocial impact of malocclusion: comparison between cleft and non-cleft patients and their parents. Eur J Orthod. 2019;41(1):38-45. https://doi.org/10.1093/ejo/cjy022

32. Nicholls W, Harper C, Selvey LA, Robinson S, Hartig G, Persson M. Body esteem in a western Australian cleft lip and/or palate cohort across 3 age groups. Cleft Palate Craniofac J. 2018;55(4):487-98. https://doi.org/10.1177/1055665617730362

33. Pisula E, Lukowska E, Fudalej PS. Self-esteem, coping styles, and quality of life in polish adolescents and young adults with unilateral cleft lip and palate. Cleft Palate Craniofac J. 2014;51(3):290-9. https://doi.org/10.1597/13-002 